hospitalisation and recovery. Somewhere in the journey, I realised that I am a person with mental illness - that it is as much a part of my identity as fatherhood or professionalism. I am proud of all of these identities. I do not want to have to keep any of them a secret by passing as normal. Instead, I expect others to join me where I stand.

1 Corrigan PW. Resolving mental illness stigma: should we seek normalcy or solidarity? Br J Psychiatry 2016; 208: 314-5.

2 Corrigan PW, Kosyluk KA, Rusch N. Reducing self-stigma by coming out proud. Am J Public Health 2013; 103: 794-800.

3 Corrigan PW, Larson JE, Hautamaki J, Matthews A, Kuwabara S, Rafacz J, et al. What lessons do coming out as gay men or lesbians have for people stigmatized by mental illness? Community Ment Health J 2009; 45 : 366-74.

4 Corrigan PW, Matthews AK. Stigma and disclosure: implications for coming out of the closet. J Ment Health 2003; 12: 235-48.

Patrick W. Corrigan, Illinois Institute of Technology. Email: corrigan@iit.edu

doi: $10.1192 / \mathrm{bjp} .209 .5 .433 a$

\section{Stephen Potts' review of To Fathom Hell or Soar Angelic}

I am saddened to see the wholly negative review of my novel, To Fathom Hell or Soar Angelic, in the June 2016 edition of the British Journal of Psychiatry. ${ }^{1}$ Obviously, I open myself up to opinions and critique when publishing anything - and especially on such a controversial subject as this - so my grievance is not about the reviewer's overall appreciation of the book, which he is obliged to state. Rather, I felt the review published in the journal was markedly unbalanced and unprofessional.

Completely disregarding the fact that the book itself is a work of fiction, and missing entirely the point about my intentional use of character stereotypes to get across the complexities of the subject, the review reads as an unnecessarily personal attack on my approach to psychiatry and medicine itself. I clearly do not hold views of contempt for psychiatry or indeed medicine, as the reviewer suggests. I have been working quite happily and successfully as a mainstream doctor for 20 years using mainstream methods. In stating otherwise, the reviewer betrays himself as irrationally fearful of exploring - or even considering - alternatives to the current medical models. It is extraordinary how a work of fiction could have stimulated such a defensive reply.

The review was riddled with misinterpretations. I object strongly to the reviewer erroneously accusing me of acting irresponsibly, by his cherry-picked and biased reporting of the facts as they appear in the book. The reviewer is forgiven for not understanding the complex pharmacology of psychedelic drugs; those of us in this field have become used to weathering such mistakes made by others regarding the risk-benefit ratio of these substances, albeit such errors are more often heard from the tabloid press than from medical professionals.

As a result of the reviewer's biased approach, he made no attempt to represent the other side of the debate regarding psychedelic drug research; rather, he simply stated his own personal opinions and used the review as platform to make his views heard. He stated his objection to the caricatured description of the novel's protagonist as a stereotypical establishment psychiatrist, yet appeared to miss entirely the balancing descriptions the book offers poking fun at the equally ridiculous drug-addled hippies. I can only assume the reviewer did not even read the book in its entirety.
I have written a number of book reviews myself over the years and I do not always agree with or necessarily like the book I am reviewing. However, I am always vigilant of the necessary guidelines around how to write a balanced review: to avoid being swayed by personal bias, to present the facts clearly and - crucially - to avoid unnecessarily inflammatory remarks. In this respect, I am surprised the review was considered to meet the usual expected standards of the journal.

1 Potts S. Book review: To Fathom Hell or Soar Angelic. Br J Psychiatry 2016; 208: $596-7$.

Ben Sessa. Email: bensessa@gmail.com

doi: 10.1192/bjp.209.5.434

Author's reply: I know from experience that negative reviews can sting, and it is tempting to lash out and shoot the messenger. I explicitly reviewed Dr Sessa's book as a work of fiction, but he objects most strongly to what he calls 'cherry-picked and biased reporting of the facts'. What should we make of 'facts' voiced by a fictional character? Dr Sessa gives no example, but on page 72, a leading character - presented as a hero - lists psychedelic drugs as 'not just LSD. Also psilocybin, MDMA, ketamine, Ibogaine ...' and goes on to say that 'they are extremely safe. They are totally physiologically non-toxic'.

If this is a fact, it is simply false: ask any emergency department doctor (or, in the case of ketamine, a urologist). Is it cherry-picking to focus on this? Any balancing statement is deeply buried. Is it irresponsible to make such an unbalanced claim about non-toxicity? In my view, yes - although I am happy to be guided to the contrary by toxicologists. Is it unprofessional to point it out in a review? I'd say it was obligatory.

On page 283 , the authorial narrator - not a character describes an identifiable National Health Service general hospital: 'A more decrepit hell-hole masquerading as a clinical setting is hard to imagine ... overflowing bags of discarded clinical waste - also known in the profession as patients - wait for collection by absent stoned porters.' I may be biased, having once worked there, but I expect the porters and professional colleagues employed at this hospital today would also see this description as contemptuous.

Dr Sessa stands by his novel. I stand by my review. Presumably, the journal stands by its decision to publish it. Perhaps we should all agree to let readers judge for themselves.

Stephen Potts. Email: stephen.g.potts@btinternet.com

doi: 10.1192/bjp.209.5.434a

\section{How much of ketamine's antidepressant response is shared with ethanol?}

In the informative review by Schoevers et al ${ }^{1}$ about ketamine's potency in the management of pain and treatment-resistant depression, the authors perceive a latent risk of ketamine misuse resulting from these treatments and forecast that misuse will become more prominent if ketamine is used broadly in clinical practice. At this juncture, it should be emphasised that acute ethanol shares some pharmacological features with ketamine, all being parts of a cascade that precipitates enhanced synaptogenesis and connectivity in cortico-limbic networks: ${ }^{2}$ non-competitive 
antagonism of glutamatergic $N$-methyl-D-aspartate (NMDA) receptors; disinhibition of pyramidal cells producing an extracellular glutamate surge; amplification of glutamate non-NMDA receptor and downstream mammalian target of rapamycin (mTOR) signalling pathways; and increase in neurotrophins (brain-derived neurotrophic factor (BDNF) and nerve growth factor (NGF) $).^{3}$ All of these are assumed to contribute to the generation of ketamine's rapid but short-term antidepressant response. $^{2}$

When ethanol vapour is repeatedly applied to rodents, their prefrontal pyramidal neurons develop an increase in dendritic spine density in the first abstinence days, ${ }^{4}$ which may resemble the synaptic remodelling observed after a single subanaesthetic ketamine pulse. $^{2}$ While the first is interpreted as reflecting plasticity changes on the way to addiction, ${ }^{4}$ the second has been shown to reverse chronic-stress-mediated decreases in spine density and is assumed to morphologically represent the antidepressant response. ${ }^{2}$ Do a few ethanol pulses work similarly, 'refreshing' on stressed spines of a non-addicted brain? Intriguingly, low ethanol doses are followed by antidepressant-like effects in Porsolt's swim test on mice. ${ }^{3}$

Against this background, there remain in my mind a few primarily depressed alcohol-dependent individuals, who reported an improvement of their depressed state after a few glasses of beer or wine. This improvement lasted for some abstinent days (ethanol's antidepressant response); however, this was only in the beginning of their drinking career. To cope with depression more sustainably, these patients gradually increased the frequency and amount of alcohol intake, which resulted in hangover and tolerance to ethanol's putative antidepressant response. Ethanol's antidepressant response might have been weaker than that of ketamine, considering ethanol's weaker antagonism of NMDA receptors and stronger stimulation of $\gamma$-aminobutyric acid (GABA) type A receptors. ${ }^{3}$ Once these patients were addicted, aversive withdrawal symptoms, craving and alcoholseeking behaviour occurred, which worsened their depression and fuelled more frequent or continuous drinking.

Abstaining alcohol-dependent individuals have lower limbic brain glutamate concentrations than normal controls, ${ }^{5}$ suggesting a long-term adaptation to too many glutamate surges alongside harmful drinking. Can this also happen to the brain when ketamine is frequently applied, thus giving birth to an aberrant learning process, such as addiction? Moreover, prolonged intake of either ethanol or ketamine is associated with gene expression of specific NMDA receptor subunits, sustained inhibition of synaptic long-term potentiation and decreasing levels of neurotrophins ${ }^{3}-$ themselves all related to an addicted brain and precursors to neurotoxicity.

Ketamine and ethanol are good examples of psychoactive drugs, whose wanted - even therapeutic - effects (e.g. the antidepressant response) can silently turn to adverse effects (e.g. addiction or neurotoxicity) after exceeding an individual critical amount and duration of intake. This is based on their ability to use the same pathway to trigger cortico-limbic plasticity involved in the generation of antidepressant response, tolerance and addiction. If at all possible, finding the optimal route of administration and dosing of ketamine to produce a preferably long-term antidepressant response without burgeoning tolerance (even to ketamine's antidepressant response) remains a big challenge. ${ }^{3}$

1 Schoevers RA, Chaves TV, Balukova SM, aan het Rot M, Kortekaas R. Oral ketamine for the treatment of pain and treatment-resistant depression. Br J Psychiatry 2016; 208: 108-13.

2 Duman $\mathrm{CH}$, Duman RS. Spine synapse remodeling in the pathophysiology and treatment of depression. Neurosci Lett 2015; 601: 20-9.

3 Bonnet $\mathrm{U}$. Long-term ketamine self-injections in major depressive disorder: focus on tolerance in ketamine's antidepressant response and the development of ketamine addiction. J Psychoactive Drugs 2015; 47: 276-85.

4 Kim A, Zamora-Martinez ER, Edwards S, Mandyam CD. Structural reorganization of pyramidal neurons in the medial prefrontal cortex of alcohol dependent rats is associated with altered glial plasticity. Brain Struct Funct 2015; 220: 1705-20.

5 Thoma R, Mullins P, Ruhl D, Monnig M, Yeo RA, Caprihan A, et al. Perturbation of the glutamate-glutamine system in alcohol dependence and remission. Neuropsychopharmacology 2011; 36: 1359-65.

Udo Bonnet, Psychiatrist, MD, Professor, Department of Psychiatry, Psychotherapy, and Psychosomatic Medicine, Evangelisches Krankenhaus Castrop-Rauxel, Germany. Email: udo.bonnet@uni-due.de

doi: $10.1192 / \mathrm{bjp} .209 .5 .434 \mathrm{~b}$

Authors' reply: We thank Professor Bonnet for his comments regarding our review on ketamine's potential for the management of pain and treatment-resistant depression. In his letter, Professor Bonnet focuses on ketamine's liability for misuse if it is broadly accepted in the clinic. He purports the idea that ketamine may have a similar liability for misuse as ethanol and backs his idea with preclinical and clinical studies showing functional changes in spine synapse remodelling and glutamatergic systems. He argues that ketamine might share some pharmacological effects with ethanol, and that such effects may eventually lead to addiction by triggering similar brain circuitry.

It is indeed true that ketamine and ethanol both relieve pain at moderate concentrations, and that both may lead to loss of consciousness at high concentrations. However, we believe that here the parallel ends, for the following reasons. First, pharmacologically, ketamine and ethanol are quite different substances; whereas ketamine isomers and their metabolites specifically bind to NMDA and aminomethylphosphonic acid (AMPA) receptors, this is very unlikely for simple molecules such as ethanol and its metabolites. Second, ethanol is a lipophilic molecule, and at higher doses it will influence GABA neurotransmission through its direct action on the chlorine channel. It might even influence cell membrane integrity at very high doses, thus indirectly influencing central neurotransmission. Third, benzodiazepines also indirectly increase GABA neurotransmission and are effective anxiolytics, but they are devoid of antidepressant effects. It can also be argued that current animal models of depression have limited value and are more likely to be measuring anxiety than depression. Fourth, in a recent Nature article, ${ }^{1}$ compelling evidence was presented that it is not ketamine itself but its $\mathrm{OH}$-norketamine metabolite that is responsible for the antidepressant effect through its action on AMPA receptors. This is also in line with earlier studies showing that ketamine has antagonistic properties at both NMDA and AMPA receptors. It was also noted that the metabolite displayed very few side-effects, which is consistent with a very specific action. ${ }^{1}$

Thus, however intriguing the suggestion of our esteemed colleague might be, we believe that any pharmacological resemblance between ketamine and ethanol is merely superficial. Still, ketamine may be responsible for addiction through its action on the reward system through dopamine $\mathrm{D}_{2}$ and serotonin $5-\mathrm{HT}_{2 \mathrm{C}}$ receptors in the ventral tegmental area.,3 We also agree that finding the optimal route of administration and dosing of ketamine to produce a preferably long-term antidepressant response without burgeoning tolerance remains a big challenge. Hopefully, the $\mathrm{OH}$-norketamine metabolite will open the door to a new generation of rapidly acting antidepressants 